Loukas Grafakos, Department of Mathematics, University of Missouri, Columbia, MO 65211, USA, email: loukas@@math.missouri.edu

\title{
LINEAR TRUNCATIONS OF THE HILBERT TRANSFORM
}

\begin{abstract}
We study $L^{p}$ to $L^{q}$ mapping properties of nonconvolution singular integral operators on $\mathbb{R}^{1}$, whose kernels are obtained by truncating the Hilbert kernel $1 / x$ in ways that depend linearly on the input variable. Some of these operators arise as special cases of the bilinear Hilbert transform and they are shown to map $L^{p}$ to $L^{q}$ for $q<p$.
\end{abstract}

\section{Introduction}

It is still unknown whether the bilinear operator with two different rates of translation

$$
H(g, f)(x)=\text { p.v. } \int_{-\infty}^{\infty} g(t-a x) f(x-t) \frac{d t}{t}
$$

maps $L^{\infty} \times L^{p}$ into $L^{p}$, for $1<p<+\infty$. Taking $g$ to be a characteristic function, gives rise to a class of operators whose $L^{p} \rightarrow L^{q}$ mapping properties are studied below.

For $a, b, d$ real numbers and $f$ a function on $\mathbb{R}^{1}$, let

$$
\left(H_{a, b, d} f\right)(x)=\int_{a x+b}^{a x+d} f(x-t) \frac{d t}{t},
$$

where the integral in (1.1) should be interpreted in the principal value sense when $(a x+b)(a x+d)<0$. Note that for $a \neq 0, H_{a, b, d}$ is not a convolution operator. When $a=1, H_{1, b, d}=H\left(f \chi_{[-d,-b]}\right)$ is the Hilbert transform composed with multiplication by the characteristic function of the interval $[-d,-b]$. When $a=0, H_{0, b, d}$ is a well understood operator. In the sequel, we will concentrate our attention to $a \neq 0,1$.

Mathematical Reviews subject classification: Primary: 42B20

Received by the editors April 12, 1996 
An easy calculation shows that $\left\langle H_{a, b, d} f, g\right\rangle=\left\langle f, H_{a, b, d}^{*} g\right\rangle$ for $f, g$ test functions, where

$$
H_{a, b, d}^{*}= \begin{cases}-H_{\frac{a}{a-1}, \frac{b}{a-1}, \frac{d}{a-1}} & \text { if } a>1 \\ -H_{\frac{a}{a-1}, \frac{d}{a-1}, \frac{b}{a-1}} & \text { if } a<1 .\end{cases}
$$

Therefore, the classes $\mathcal{L}_{1}=\left\{ \pm H_{a, b, d}: 0 \neq a<1\right\}$ and $\mathcal{L}_{2}=\left\{ \pm H_{a, b, d}: a>\right.$ 1\} are preserved under the map $T \longrightarrow T^{*}$. As we shall see, the operators in $\mathcal{L}_{1}$ and $\mathcal{L}_{2}$ map $L^{p}$ into $L^{q}$ for $1 \leq q<p \leq \infty$. This is of course naturally impossible for convolution operators, which must be identically equal to zero if they have such a property. See [StW, page 33, 4.11].

Let us introduce some notation. $L^{p}$ will always be $L^{p}\left(\mathbb{R}^{1}\right)$. For a function $f(x)$ on $\mathbb{R}^{1}, \tau \in \mathbb{R}$, and $\delta>0, f_{\delta}(x)$ will denote the function $f(\delta x), \tilde{f}(x)$ the function $f(-x)$, and $\tau f(x)$ the function $f(x+\tau)$. In this paper the upper and lower limits of our integrals are allowed to be arbitrary real numbers and all integrals are interpreted in the principle value sense when necessary. Throughout, $C_{k, l, m}$ will be a generic positive constant depending on the parameters $k, l$, and $m$.

Since for $a \neq 1 H_{a, b, d}$ is not a convolution operator, it doesn't commute with translations. Nevertheless, it commutes with dilations in the following sense:

$$
H_{a, b, d}\left(f_{\delta}\right)=\left(H_{a, \delta b, \delta d} f\right)_{\delta} .
$$

The main result concerning $H_{a, b, d}$ is the following:

Theorem 1 Let $a, p, q$ be real numbers satisfying $a \neq 0,1<p \leq \infty$, $1 \leq q \leq p$, and $q<\infty$. Then, the operator $H_{a, b, d}$ maps $L^{p}$ to $L^{q}$ with norm $\leq C_{p, q, a}|b-d|^{\frac{1}{q}-\frac{1}{p}}$.

Next, we consider slightly different truncations of the Hilbert kernel. Let

$$
\left(I_{a, b, d} f\right)(x)=\int_{a|x|+b}^{a|x|+d} f(x-t) \frac{d t}{t},
$$

where (1.4) should be interpreted in the principal value sense if $(a|x|+b)(a|x|+$ $d)<0$. We have the following:

Theorem 2 Let $a, p$, and $q$ be as before. Then $I_{a, b, d}$ maps $L^{p}$ to $L^{q}$ with norm $\leq C_{p, q, a}|b-d|^{\frac{1}{q}-\frac{1}{p}}$. 
Finally, for $a \neq 0$, and $c \neq 0$, we define the operators

$$
\begin{aligned}
\left(H_{a, b, c, d} f\right)(x) & =\int_{a x+b}^{c x+d} f(x-t) \frac{d t}{t} \text { and } \\
\left(I_{a, b, c, d} f\right)(x) & =\int_{a|x|+b}^{c|x|+d} f(x-t) \frac{d t}{t},
\end{aligned}
$$

where the integrals above are taken in the principal value sense when it is appropriate. Surprisingly, these two classes of operators behave very differently when $a \neq c$. An example in Section 6 shows that $H_{a, b, c, d}$ may not map $L^{p}$ to any $L^{q}$. However, we have the following Theorem regarding $I_{a, b, c, d}$ :

Theorem 3 When $a, c$ are nonzero, the operator $I_{a, b, c, d}$ maps $L^{p}$ to $L^{p}$, for $1<p<+\infty$.

In general, the operators $I_{a, b, c, d}$ do not map $L^{p}$ to $L^{q}$ for $q \neq p$, nor $L^{p} \rightarrow L^{p}$ for $p=1$ or $\infty$. Examples are given in Section 5. So Theorem 3 is sharp in this sense.

The main tool in the proof of the Theorems above is the following Lemma, which is a variation of a result of Hardy. Its proof is postponed until Section 6 (appendix).

Lemma For every $1<p<\infty$ and every non-zero real $\alpha$ there exists a positive constant $C_{p, \alpha}$ such that for all $f \in L^{p}\left(\mathbb{R}^{1}\right)$ the operator

$$
\left(T_{\alpha} f\right)(x)=\int_{\alpha x}^{+\infty} f(t-x) \frac{d t}{t}
$$

satisfies:

(i) $\left\|T_{\alpha} f\right\|_{L^{p}(0, \infty)} \leq C_{p, \alpha}\|f\|_{L^{p}}$ if $\alpha>0$ and

(ii) $\left\|T_{\alpha} f\right\|_{L^{p}(-\infty, 0)} \leq C_{p, \alpha}\|f\|_{L^{p}}$ if $\alpha<0$.

We break the proof of Theorem 1 in the following three cases which are treated in Sections 2, 3, and 4 respectively:
A. $1<p<\infty, \quad 1 \leq q \leq p, \quad$ and $0<a<1$.
B. $p=\infty, \quad 1 \leq q<\infty$, and $a>0,(a \neq 1)$.
C. $1<p<\infty, \quad 1 \leq q \leq p, \quad$ and $a>1$.

Once these three cases have been established, duality, (1.2), and the fact that the map $a \longrightarrow \frac{a}{a-1}$ is a bijection between $(0,1)$ and $(-\infty, 0)$ can be used to show that Theorem 1 also holds for $a<0$, (and thus to complete its proof). 
2 The Case $1<p<\infty, 1 \leq q \leq p$, and $0<a<1$

Fix $p, q$ and $a$ as indicated. We are going to show that there exists a constant $C_{p, q, a}$ such that

$$
\left\|H_{a, b, d} f\right\|_{L^{q}} \leq C_{p, q, a}|b-d|^{\frac{1}{q}-\frac{1}{p}}\|f\|_{L^{p}} .
$$

(2.1) will follow by interpolation from

$$
\left\|H_{a, b, d} f\right\|_{L^{1}} \leq C_{p, a}|b-d|^{\frac{1}{p^{\prime}}}\|f\|_{L^{p}}
$$

and

$$
\left\|H_{a, b, d} f\right\|_{L^{p}} \leq C_{p, a}\|f\|_{L^{p}} .
$$

Without loss of generality, we may assume that $d>b$. Also, a simple dilation argument and (1.3) show that it suffices to prove (2.2) and (2.3) with $d-b=1$. We therefore assume that $d-b=1$. Changing variables, we write

$$
\left(H_{a, b, d} f\right)(x)=\int_{(1-a) x-d}^{(1-a) x-b} \frac{f(t)}{x-t} d t .
$$

We consider the following three cases:

Case $1: x>-\frac{b}{a}$

Case $2: x<-\frac{d}{a}$

Case $3:-\frac{d}{a} \leq x \leq-\frac{b}{a}$

We start with case 1 . Note that if $x>-\frac{b}{a}$, the quantity $x-t$ in (2.4) is positive. Using (2.4) we obtain

$$
\begin{aligned}
\int_{-\frac{b}{a}}^{+\infty} & \left|\left(H_{a, b, d} f\right)(x)\right| d x \leq \int_{-\frac{b}{a}}^{+\infty} \int_{(1-a) x-d}^{(1-a) x-b}|f(t)| \frac{d t}{x-t} d x \\
& =\int_{-\frac{b}{a}-1}^{-\frac{b}{a}}|f(t)| \int_{-\frac{b}{a}}^{\frac{t+d}{1-a}} \frac{d x}{x-t} d t+\int_{-\frac{b}{a}}^{+\infty}|f(t)| \int_{\frac{t+b}{1-a}}^{\frac{t+d}{1-a}} \frac{d x}{x-t} d t \quad \text { by Fubini } \\
& =\int_{-1}^{0}\left|f\left(t-\frac{b}{a}\right)\right| \ln \left|\frac{a}{1-a} \frac{t+\frac{1}{a}}{t}\right| d t+\int_{0}^{+\infty}\left|f\left(t-\frac{b}{a}\right)\right| \ln \left|\frac{t+\frac{1}{a}}{t}\right| d t \\
& =I+I I .
\end{aligned}
$$


We now estimate $I$ and $I I$. For $1<p<\infty$, set

$$
B_{p}=\left\|\ln \left|1+\frac{1}{x}\right|\right\|_{L^{p}(-\infty,+\infty)}<+\infty .
$$

By Hölder's inequality we have

$$
I I \leq\|f\|_{L^{p}}\left\{\int_{0}^{\infty}|\ln | 1+\frac{1}{t a}||^{p^{\prime}} d t\right\}^{\frac{1}{p^{\prime}}}=B_{p^{\prime}} a^{-\frac{1}{p^{\prime}}}\|f\|_{L^{p}}
$$

and

$$
\begin{aligned}
I & \leq\|f\|_{L^{p}}\left\{\int_{-1}^{0}|\ln | \frac{a}{1-a}\left(1+\frac{1}{a t}\right) \|^{p^{\prime}} d t\right\}^{\frac{1}{p^{\prime}}} \\
& =\|f\|_{L^{p}} a^{-\frac{1}{p^{\prime}}}\left\{\int_{-a}^{0}|\ln | \frac{a}{1-a}|+\ln | 1+\frac{1}{t}||^{p^{\prime}} d t\right\}^{\frac{1}{p^{\prime}}} \\
& \leq\|f\|_{L^{p}} a^{-\frac{1}{p^{\prime}}}\left\{a^{\frac{1}{p^{\prime}}}|\ln | \frac{a}{1-a}||+B_{p^{\prime}}\right\} .
\end{aligned}
$$

(2.5) and (2.6) now imply

$$
\int_{-\frac{b}{a}}^{+\infty}\left|\left(H_{a, b, d} f\right)(x)\right| d x \leq C_{p, a}\|f\|_{L^{p}} .
$$

To obtain the $L^{p}$ estimate in this case, note that for $x>-\frac{b}{a}$

$$
\begin{aligned}
& \left|\left(H_{a, b, d} f\right)(x)\right| \leq \int_{a x+b}^{a x+d}|f(x-t)| \frac{d t}{t} \leq \int_{a\left(x+\frac{b}{a}\right)}^{+\infty}|f(x-t)| \frac{d t}{t} \\
& =\int_{a\left(x+\frac{b}{a}\right)}^{+\infty}\left|\tilde{f}\left(\frac{b}{a}+t-\left(x+\frac{b}{a}\right)\right)\right| \frac{d t}{t}=T_{a}\left(\left.\right|_{\frac{b}{a}} \tilde{f} \mid\right)\left(x+\frac{b}{a}\right)==_{\frac{b}{a}}\left(T_{a}\left(\left.\right|_{\frac{b}{a}} \tilde{f} \mid\right)\right)(x),
\end{aligned}
$$

which maps $L^{p}$ to $L^{p}\left(-\frac{b}{a}, \infty\right)$ by the Lemma. We conclude that

$$
\left(\int_{-\frac{b}{a}}^{+\infty}\left|H_{a, b, d} f\right|^{p} d x\right)^{\frac{1}{p}} \leq C_{p, a}\|f\|_{L^{p}} .
$$


We proceed with case 2 . Note that if $x<-\frac{d}{a}$, the quantity $x-t$ in (2.4) is negative. Using (2.4) we have

$$
\begin{aligned}
\int_{-\infty}^{-\frac{d}{a}}\left|\left(H_{a, b, d} f\right)(x)\right| d x \leq \int_{-\infty}^{-\frac{d}{a}} \int_{(1-a) x-d}^{(1-a) x-b}|f(t)| \frac{d t}{t-x} d x \\
=\int_{-\infty}^{-\frac{d}{a}}|f(t)| \int_{\frac{t+b}{1-a}}^{\frac{t+d}{1-a}} \frac{d x}{t-x} d t+\int_{-\frac{d}{a}}^{-\frac{d}{a}+1}|f(t)| \int_{\frac{t+b}{1-a}}^{-\frac{d}{a}} \frac{d x}{t-x} d t \quad \text { by Fubini } \\
=\int_{-\infty}^{0}\left|f\left(t-\frac{d}{a}\right)\right| \ln \left|\frac{t-\frac{1}{a}}{t}\right| d t+\int_{0}^{1}\left|f\left(t-\frac{d}{a}\right)\right| \ln \left|\frac{a}{a-1} \frac{t-\frac{1}{a}}{t}\right| d t \\
\leq 2\|f\|_{L^{p}} a^{-\frac{1}{p^{\prime}}} B_{p^{\prime}}+\|f\|_{L^{p}}|\ln | \frac{a}{a-1}||=C_{p, a}\|f\|_{L^{p}} .
\end{aligned}
$$

Also note that for $x<-\frac{d}{a}$

$$
\begin{aligned}
\left|\left(H_{a, b, d} f\right)(x)\right| & \leq \int_{a x+b}^{a x+d}|f(x-t)| \frac{d t}{|t|}=\int_{-a x-d}^{-a x-b}|f(x+t)| \frac{d t}{t} \\
& \leq \int_{a\left(-x-\frac{d}{a}\right)}^{+\infty}|f(x+t)| \frac{d t}{t}=\int_{a\left(-x-\frac{d}{a}\right)}^{+\infty}\left|f\left(-\frac{d}{a}+t-\left(-x-\frac{d}{a}\right)\right)\right| \frac{d t}{t} \\
& =T_{a}\left(\left.\right|_{-\frac{d}{a}} f \mid\right)\left(-x-\frac{d}{a}\right)={ }_{-\frac{d}{a}}\left(T_{a} \widehat{\left.\left(\left.\right|_{-\frac{d}{a}} f \mid\right)\right)}(x),\right.
\end{aligned}
$$

which maps $L^{p}$ to $L^{p}\left(-\infty,-\frac{d}{a}\right)$. We therefore obtain

$$
\left(\int_{-\infty}^{-\frac{d}{a}}\left|H_{a, b, d} f\right|^{p} d x\right)^{\frac{1}{p}}+\int_{-\infty}^{-\frac{d}{a}}\left|H_{a, b, d} f\right| d x \leq C_{p, a}\|f\|_{L^{p}} .
$$

Case 3 is different. For $-\frac{d}{a} \leq x \leq-\frac{b}{a}$, we write

$$
\left(H_{a, b, d} f\right)(x)=(H f)(x)-\int_{-\infty}^{a x+b} f(x-t) \frac{d t}{t}-\int_{a x+d}^{+\infty} f(x-t) \frac{d t}{t}
$$


Since

$$
\|H f\|_{L^{1}\left[-\frac{d}{a},-\frac{b}{a}\right]} \leq a^{-\frac{1}{p^{\prime}}}\|H f\|_{L^{p}\left[-\frac{d}{a},-\frac{b}{a}\right]} \leq a^{-\frac{1}{p^{\prime}}} C_{p}\|f\|_{L^{p}}
$$

it suffices to bound the $L^{1}$ and $L^{p}$ norms on $\left[-\frac{d}{a},-\frac{b}{a}\right]$ of the other two terms in (2.10). We now estimate these terms.

$$
\begin{aligned}
\left|\int_{a x+d}^{+\infty} f(x-t) \frac{d t}{t}\right| & \leq \int_{a x+d}^{+\infty}|f(x-t)| \frac{d t}{t}=\int_{a\left(x+\frac{d}{a}\right)}^{+\infty}|\tilde{f}(t-x)| \frac{d t}{t} \\
& =\int_{a\left(x+\frac{d}{a}\right)}^{+\infty}\left|\tilde{f}\left(\frac{d}{a}+t-\left(x+\frac{d}{a}\right)\right)\right| \frac{d t}{t}=T_{a}\left(\left.\right|_{\frac{d}{a}} \tilde{f} \mid\right)\left(x+\frac{d}{a}\right) \\
& =\frac{d}{a}\left(T_{a}\left(\left.\right|_{\frac{d}{a}} \tilde{f} \mid\right)\right)(x)
\end{aligned}
$$

Also

$$
\begin{aligned}
& \left|\int_{-\infty}^{a x+b} f(x-t) \frac{d t}{t}\right|=\left|\int_{-a x-b}^{+\infty} f(x+t) \frac{d t}{t}\right| \leq \int_{a\left(-x-\frac{b}{a}\right)}^{+\infty}|f(x+t)| \frac{d t}{t} \\
& =\int_{a\left(-x-\frac{b}{a}\right)}^{+\infty}\left|f\left(-\frac{b}{a}+t-\left(-x-\frac{b}{a}\right)\right)\right| \frac{d t}{t}=T_{a}\left(\left.\right|_{-\frac{b}{a}} f \mid\right)\left(-x-\frac{b}{a}\right) \\
& ={ }_{-\frac{b}{a}}\left(T_{a}\left(\left.\right|_{-\frac{b}{a}} f \mid\right)\right)(x)
\end{aligned}
$$

An application of the Lemma gives the following $L^{p}$ estimate

$$
\left\|_{\frac{d}{a}}\left(T_{a}\left(\left.\right|_{\frac{d}{a}} \tilde{f} \mid\right)\right)\right\|_{L^{p}\left[-\frac{d}{a},-\frac{b}{a}\right]} \leq\left\|T_{a}\left(\left.\right|_{\frac{d}{a}} \tilde{f} \mid\right)\right\|_{L^{p}[0, \infty)} \leq C_{p, a}\|f\|_{L^{p}}
$$

and consequently the $L^{1}$ estimate

$$
\begin{aligned}
\left\|_{\frac{d}{a}}\left(T_{a}\left(\left.\right|_{\frac{d}{a}} \tilde{f} \mid\right)\right)\right\|_{L^{1}\left[-\frac{d}{a},-\frac{b}{a}\right]} & \leq a^{-\frac{1}{p^{\prime}}}\left\|_{\frac{d}{a}}\left(T_{a}\left(\left.\right|_{\frac{d}{a}} \tilde{f} \mid\right)\right)\right\|_{L^{p}\left[-\frac{d}{a},-\frac{b}{a}\right]} \\
& \leq C_{p, a}\|f\|_{L^{p}}
\end{aligned}
$$

Similarly, we obtain

$$
\begin{aligned}
\left.\|_{-\frac{b}{a}}\left(\widetilde{T_{a}\left(\left.\right|_{-\frac{b}{a}} f\right.} \mid\right)\right) \|_{L^{p}\left[-\frac{d}{a},-\frac{b}{a}\right]} & \leq\left\|T_{a}\left(\left.\right|_{-\frac{b}{a}} f \mid\right)\right\|_{L^{p}[0, \infty)} \\
& \leq C_{p, a}\|f\|_{L^{p}}
\end{aligned}
$$


and the corresponding $L^{1}$ estimate

$$
\begin{aligned}
\left.\|_{-\frac{b}{a}}\left(T_{a} \widetilde{\left(\left.\right|_{-\frac{b}{a}} f\right.} \mid\right)\right) \|_{L^{1}\left[-\frac{d}{a},-\frac{b}{a}\right]} & \left.\leq a^{-\frac{1}{p^{\prime}}} \|_{-\frac{b}{a}}\left(T_{a} \widetilde{\left(\left.\right|_{-\frac{b}{a}} f\right.} \mid\right)\right) \|_{L^{p}\left[-\frac{d}{a},-\frac{b}{a}\right]} \\
& \leq C_{p, a}\|f\|_{L^{p}} .
\end{aligned}
$$

Using (2.10)-(2.17) we conclude that for $0<a<1$

$$
\left\|H_{a, b, d} f\right\|_{L^{1}\left[-\frac{d}{a}-\frac{b}{a}\right]}+\left\|H_{a, b, d} f\right\|_{L^{p}\left[-\frac{d}{a},-\frac{b}{a}\right]} \leq C_{p, a}\|f\|_{L^{p}} .
$$

(2.7), (2.8), (2.9), and (2.18) now imply

$$
\left\|H_{a, b, d} f\right\|_{L^{1}}+\left\|H_{a, b, d} f\right\|_{L^{p}} \leq C_{p, a}\|f\|_{L^{p}},
$$

which completes the proof of (2.2) and (2.3).

An application of the M. Riesz-Thorin interpolation Theorem ([Z, p. 95]), gives the required estimate (2.1).

\section{The Case $p=\infty, \quad 1 \leq q<\infty$, and $a>0(a \neq 1)$}

Fix $a, p$ and $q$ as indicated. As in the previous section, we can reduce matters to the situation when $d-b=1$. We must show that

$$
\left\|H_{a, b, d} f\right\|_{L^{q}} \leq C_{a, q}\|f\|_{L^{\infty}} .
$$

We consider the same three cases as in Section 2.

For $x>-\frac{b}{a}$ we have

$\left|\left(H_{a, b, d} f\right)(x)\right| \leq \int_{a x+b}^{a x+d}|f(x-t)| \frac{d t}{t} \leq\|f\|_{L^{\infty}} \ln \left|\frac{a x+d}{a x+b}\right|=\|f\|_{L^{\infty}} \ln \left|\frac{x+\frac{b}{a}+\frac{1}{a}}{x+\frac{b}{a}}\right|$

and since obviously

$$
\int_{-\frac{b}{a}}^{+\infty}|\ln | \frac{x+\frac{b}{a}+\frac{1}{a}}{x+\frac{b}{a}}||^{q} d x \leq \frac{B_{q}^{q}}{a}<+\infty
$$

it follows that

$$
\left\|H_{a, b, d} f\right\|_{L^{q}\left(-\frac{b}{a}, \infty\right)} \leq a^{-\frac{1}{q}} B_{q}\|f\|_{L^{\infty}} .
$$


Fox $x<-\frac{d}{a}$ we have

$$
\left|\left(H_{a, b, d}\right)(x)\right| \leq \int_{a x+b}^{a x+d}|f(x-t)| \frac{d t}{-t} \leq\|f\|_{L^{\infty}} \ln \left|\frac{x+\frac{d}{a}-\frac{1}{a}}{x+\frac{d}{a}}\right|
$$

and since

$$
\int_{-\infty}^{-\frac{d}{a}}|\ln | \frac{x+\frac{d}{a}-\frac{1}{a}}{x+\frac{d}{a}}||^{q} d x \leq \frac{B_{q}^{q}}{a}<+\infty
$$

we obtain

$$
\left\|H_{a, b, d} f\right\|_{L^{q}\left(-\infty,-\frac{d}{a}\right)} \leq a^{-\frac{1}{q}} B_{q}\|f\|_{L^{\infty}} .
$$

Finally for $-\frac{d}{a} \leq x \leq-\frac{b}{a}$ we argue as follows: Observe that for $a x+b \leq t \leq$ $a x+d$ we have $-a x-d-\frac{d}{a} \leq x-t \leq-a x-b-\frac{b}{a}$ and thus $b-d-\frac{d}{a} \leq$ $x-t \leq d-b-\frac{b}{a}$. We have

$$
\left(H_{a, b, d}\right)(x)=\int_{a x+b}^{a x+d} f(x-t) \frac{d t}{t}=\int_{a x+b}^{a x+d}\left(f \chi_{\left[-1-\frac{d}{a}, 1-\frac{b}{a}\right]}\right)(x-t) \frac{d t}{t} .
$$

It follows from (2.18) that

$$
\begin{aligned}
\left\|H_{a, b, d} f\right\|_{L^{q}\left[-\frac{d}{a},-\frac{b}{a}\right]} & \leq C_{q, a}\left\|f \chi_{\left[-1-\frac{d}{a}, 1-\frac{b}{a}\right]}\right\|_{L^{q}} \\
& \leq C_{q, a}\left(2+\frac{1}{a}\right)^{\frac{1}{q}}\|f\|_{L^{\infty}}=C_{q, a}^{\prime}\|f\|_{L^{\infty}}
\end{aligned}
$$

We conclude from (3.2), (3.3), and (3.4) that (3.1) holds.

\section{The Case $1<p<\infty, 1 \leq q \leq p$, and $a>1$}

Fix $a, p$ and $q$ as indicated. As before we assume that $d-b=1$ and consider the same three cases as in Section 2. Observing that $x-t>0$ in (2.4) when $x>-\frac{b}{a}$, we have

$$
\int_{-\frac{b}{a}}^{+\infty}\left|\left(H_{a, b, d} f\right)(x)\right| d x \leq \int_{-\frac{b}{a}}^{+\infty} \int_{(1-a) x-d}^{(1-a) x-b}|f(t)| \frac{d t}{x-t} d x
$$




$$
\begin{aligned}
& =\int_{-\frac{b}{a}}^{+\infty} \int_{(a-1) x+b}^{(a-1) x+d}|f(-t)| \frac{d t}{x+t} d x \\
& =\int_{\frac{b}{a}}^{1+\frac{b}{a}}|\tilde{f}(t)| \int_{-\frac{b}{a}}^{\frac{t-b}{a-1}} \frac{d x}{x+t} d t+\int_{1+\frac{b}{a}}^{+\infty}|\tilde{f}(t)| \int_{\frac{t-d}{a-1}}^{\frac{t-b}{a-1}} \frac{d x}{x+t} d t \quad \text { by Fubini } \\
& =\int_{0}^{1}\left|\tilde{f}\left(t+\frac{b}{a}\right)\right| \ln \left|\frac{a}{a-1}\right| d t+\int_{1}^{+\infty}\left|\tilde{f}\left(t+\frac{b}{a}\right)\right| \ln \left|\frac{t}{t-\frac{1}{a}}\right| d t .
\end{aligned}
$$

The function $\ln \left|\frac{t}{t-\frac{1}{a}}\right| \in L^{p^{\prime}}$. Controlling (4.1) using Hölder's inequality, we obtain:

$$
\int_{-\frac{b}{a}}^{+\infty}\left|\left(H_{a, b, d} f\right)(x)\right| d x \leq\left(\ln \left|\frac{a}{a-1}\right|+a^{-\frac{1}{p^{\prime}}} B_{p^{\prime}}\right)\|f\|_{L^{p}} .
$$

When $x<-\frac{d}{a}$, the expression $x-t$ in (2.4) is negative. We then have

$$
\begin{aligned}
\int_{-\infty}^{-\frac{d}{a}} \mid & \left(H_{a, b, d} f\right)(x)\left|d x \leq \int_{-\infty}^{-\frac{d}{a}} \int_{(1-a) x-d}^{(1-a) x-b}\right| f(t) \mid \frac{d t}{t-x} d x \\
= & \int_{-\infty}^{-\frac{d}{a}} \int_{(a-1) x+b}^{(a-1) x+d}|f(-t)| \frac{d t}{-x-t} d x \\
= & \int_{-\infty}^{\frac{d}{a}-1}|\tilde{f}(t)| \int_{-\infty}^{\frac{t-b}{a-1}} \frac{d x}{-x-t} d t+\int_{\frac{t-d}{a-1}}^{\frac{d}{a}-1}|\tilde{f}(t)| \int_{-\infty}^{\frac{d}{a}} \frac{d x}{-x-t} d t \quad \text { by Fubini } \\
= & \int_{-\infty}^{-1}\left|\tilde{f}\left(t+\frac{d}{a}\right)\right| \ln \left|\frac{t}{t+\frac{1}{a}}\right| d t+\int_{-1}^{0}\left|\tilde{f}\left(t+\frac{d}{a}\right)\right| \ln \left|\frac{a}{a-1}\right| d t
\end{aligned}
$$

As observed, the function $\ln \left|\frac{t}{t+\frac{1}{a}}\right|$ is in $L^{p^{\prime}}$. By Hölder's inequality, (4.3) is bounded by

$$
\left(a^{-\frac{1}{p^{\prime}}} B_{p^{\prime}}+\ln \left|\frac{a}{a-1}\right|\right)\|f\|_{L^{p}}
$$


Adding (4.2) and (4.4) we obtain

$$
\int_{-\frac{b}{a}}^{+\infty}\left|\left(H_{a, b, d} f\right)(x)\right| d x+\int_{-\infty}^{-\frac{d}{a}}\left|\left(H_{a, b, d} f\right)(x)\right| d x \leq C_{p, a}\|f\|_{L^{p}} .
$$

The corresponding $L^{p}$ inequalities for cases 1 and 2,

$$
\left\|H_{a, b, d} f\right\|_{L^{p}\left(-\frac{b}{a}, \infty\right)}+\left\|H_{a, b, d} f\right\|_{L^{p}\left(-\infty,-\frac{d}{a}\right)} \leq C_{p, a}\|f\|_{L^{p}},
$$

as well as the estimates for case 3 ,

$$
\left\|H_{a, b, d} f\right\|_{L^{p}\left[-\frac{d}{a},-\frac{b}{a}\right]}+\left\|H_{a, b, d} f\right\|_{L^{1}\left[-\frac{d}{a}-\frac{b}{a}\right]} \leq C_{p, a}\|f\|_{L^{p}},
$$

are obtained as in Section 2. Combining (4.5), (4.6), and (4.7) we obtain the required result.

\section{Comments on Theorems 2 and 3}

The proof of the $L^{p} \rightarrow L^{q}$ boundedness of $I_{a, b, d}$ is similar to that of $H_{a, b, d}$. The only point of deviation is that one needs to consider the cases $x \geq 0$ and $x<0$ separately. The details are omitted.

The following example shows that the first operator in (1.5) may not map $L^{p}$ to $L^{q}$. Let $f_{L}=\chi_{[-L, 0]}$ where $L>0$. A simple calculation shows that $H_{1,0,2,0} f_{L}$ is identically equal to $\ln 2$ on the interval $(-\infty, L]$. This makes it impossible for $H_{1,0,2,0} f_{L}$ to belong to any Lebesgue space.

We now discuss the proof of Theorem 3. Since the line of ideas of its proof is similar to that of Theorem 1, we are going to be sketchy.

We consider the cases $x \geq 0$ and $x<0$ separately. We begin with the case $x \geq 0$. We have the following three subcases:

(i) $x \geq 0$ and $x>\max \left(-\frac{b}{a},-\frac{d}{c}\right)$;

(ii) $x \geq 0$ and $\min \left(-\frac{b}{a},-\frac{d}{c}\right) \leq x \leq \max \left(-\frac{b}{a},-\frac{d}{c}\right)$;

(iii) $x \geq 0$ and $x<\min \left(-\frac{b}{a},-\frac{d}{c}\right)$.

In subcases (i) and (iii) $I_{a, b, c, d} f$ can be estimated by the operator $T_{\alpha}$ of the Lemma applied to a translate of $f$ or its reflection $\tilde{f}$, as in Section 2. In subcase (ii) write

$$
\left(H_{a, b, c, d} f\right)(x)=(H f)(x)-\int_{-\infty}^{m} f(x-t) \frac{d t}{t}-\int_{M}^{+\infty} f(x-t) \frac{d t}{t}
$$


where $M=\max (a x+b, c x+d)$ and $m=\min (a x+b, c x+d)$. Note that in this case $t$ doesn't change sign in the integrals above. Therefore, the second and third terms in (5.1) can be estimated using the Lemma in Section 1. We omit the details.

When $x$ is negative, we have the following three subcases:

(i) $x<0$ and $x>\max \left(\frac{b}{a}, \frac{d}{c}\right)$;

(ii) $x<0$ and $\min \left(\frac{b}{a}, \frac{d}{c}\right) \leq x \leq \max \left(\frac{b}{a}, \frac{d}{c}\right)$;

(iii) $x<0$ and $x<\min \left(\frac{b}{a}, \frac{d}{c}\right)$.

In subcases (i) and (iii) we apply the Lemma directly and in subcase (ii) we use (5.1), the boundedness of the Hilbert transform on $L^{p}$, and the Lemma.

Finally we show that $I_{a, b, c, d}$ may not map $L^{p}$ to $L^{q}$ when $p \neq q$. Let $f_{L}=\chi_{(-\infty, L]}$ as before and consider the operator $I_{1,0,2,0}$. An easy calculation shows that $I_{1,0,2,0} f_{L}$ is equal to the constant $\ln 2$ on the interval $\left[-\frac{L}{3}, L\right]$ and to $\ln \left|\frac{L+x}{x}\right|$ outside this interval. If $I_{1,0,2,0}$ mapped $L^{p}$ to $L^{q}$, the inequality $\left\|I_{1,0,2,0} f_{L}\right\|_{L^{q}} \leq C_{p, q}\left\|f_{L}\right\|_{L^{p}}$ would imply $L^{\frac{1}{q}} \leq C_{p, q}^{\prime} L^{\frac{1}{p}}$ for some $C_{p, q}^{\prime}>0$. This can hold for all $L>0$ only when $p=q$. Also, considering $I_{1,0,2,0}$ and $I_{1,1,2,0}$ we conclude that boundedness from $L^{1} \rightarrow L^{1}$ and $L^{\infty} \rightarrow L^{\infty}$ holds sometimes, but not always in Theorem 3 .

\section{Appendix. Proof of the Lemma}

For $\alpha=1$ this is a known Lemma due to Hardy. See for instance [HLP, Theorem 319] or [S, appendix A3]. Its proof is based on the estimate:

$$
\left|\left(T_{1} f\right)(x)\right| \leq \int_{0}^{+\infty}|f(t x)| \frac{d t}{1+t}=\int_{0}^{+\infty}|f(t x)| t^{\frac{1}{p}} \frac{d t}{(1+t) t^{\frac{1}{p}}} .
$$

Then Minkowski's integral inequality gives

$$
\left\|T_{1} f\right\|_{L^{p}(0, \infty)} \leq \int_{0}^{+\infty} \frac{d t}{(1+t) t^{\frac{1}{p}}}\|f\|_{L^{p}(0, \infty)} \leq C_{p}\|f\|_{L^{p}} .
$$

The case $\alpha>1$ is a consequence of the case $\alpha=1$, in view of the fact that

$$
\left|\int_{\alpha x}^{+\infty} f(t-x) \frac{d t}{t}\right| \leq \int_{x}^{+\infty}|f(t-x)| \frac{d t}{t} .
$$


We now do the case $0<\alpha<1$.

$$
\begin{aligned}
\left|\int_{\alpha x}^{+\infty} f(t-x) \frac{d t}{t}\right| & \leq \int_{\alpha x}^{+\infty}|f(t-x)| \frac{d t}{t}=\int_{(\alpha-1) x}^{+\infty}|f(t)| \frac{d t}{t+x} \\
& =\int_{0}^{(1-\alpha) x}|f(-t)| \frac{d t}{x-t}+\int_{0}^{+\infty}|f(t)| \frac{d t}{t+x} \\
& =\int_{0}^{1-\alpha}|f(-t x)| \frac{d t}{1-t}+\int_{0}^{+\infty}|f(t x)| \frac{d t}{t+x}=I+I I .
\end{aligned}
$$

As before the $L^{p}(0, \infty)$ norm of term $I I$ is bounded by $C_{p}\|f\|_{L^{p}(0, \infty)}$. Now

$$
I=\int_{0}^{1-\alpha}|f(-t x)| t^{\frac{1}{p}} \frac{d t}{t^{\frac{1}{p}(1-t)}},
$$

and another application of Minkowski's integral inequality gives that the $L^{p}(0, \infty)$ norm of $I$ is bounded by

$$
\|f\|_{L^{p}(-\infty, 0)} \int_{0}^{1-\alpha} \frac{d t}{t^{\frac{1}{p}}(1-t)} \leq C_{p, \alpha}\|f\|_{L^{p}} .
$$

Part (i) of the Lemma is proved.

We now prove part (ii). For $\alpha<0$ and $x<0$ we have

$$
\begin{aligned}
\left|\int_{\alpha x}^{+\infty} f(t-x) \frac{d t}{t}\right| & \leq \int_{\alpha x}^{+\infty}|f(t-x)| \frac{d t}{t}=\int_{(\alpha-1) x}^{+\infty}|f(t)| \frac{d t}{t+x} \\
& =\int_{(\alpha-1) x}^{+\infty}|f(t)| \frac{d t}{t-1}=\int_{1-\alpha}^{+\infty}|f(-x t)| t^{\frac{1}{p}} \frac{d t}{t^{\frac{1}{p}(t-1)}} .
\end{aligned}
$$

Since $1-\alpha>1$, the function $t^{-\frac{1}{p}}(t-1)^{-1}$ is integrable on $[1-\alpha, \infty)$ and Minkowski's integral inequality will give the desired conclusion.

The following $n^{\text {th }}$ dimensional analogue of the Lemma in discussion can be obtained similarly. This was independently observed by [SoW, Lemma 2]. 
Remark Let $\alpha>0$. For a function $f$ on $\mathbb{R}^{n}$, let

$$
\left(T_{\alpha} f\right)(x)=\int_{\mathbb{R}^{n}} f(y) \frac{d y}{|y|^{n}+\alpha^{n}|x|^{n}} .
$$

Then $T_{\alpha}$ maps $L^{p}\left(\mathbb{R}^{n}\right)$ into itself for $1<p<\infty$.

Proof. Indeed, changing variables and using polar coordinates, we write

$$
\begin{aligned}
(T f)(x) & =\int_{\mathbb{R}^{n}} f(|x| y) \frac{d y}{|y|^{n}+\alpha^{n}} \\
& =\int_{r=0}^{+\infty}\left\{\int_{S^{n-1}} f(r|x| \theta) r^{\frac{n}{p}} d \theta\right\} \frac{r^{n-1} d r}{r^{\frac{n}{p}}\left(r^{n}+\alpha^{n}\right)} .
\end{aligned}
$$

Taking $L^{p}$ norms (in $x$ ) and using Minkowski's integral inequality, we obtain

$\|T f\|_{L^{p}\left(\mathbb{R}^{n}\right)} \leq \omega_{n-1}\left(\int_{r=0}^{+\infty} \frac{r^{n-1} d r}{r^{\frac{n}{p}}\left(r^{n}+\alpha^{n}\right)}\right)\|f\|_{L^{p}\left(\mathbb{R}^{n}\right)}=C_{p, n, \alpha}\|f\|_{L^{p}\left(\mathbb{R}^{n}\right)}$,

where $\omega_{n-1}$ denotes the area of the unit sphere $S^{n-1} \subset \mathbb{R}^{n}$. This finishes the proof.

Finally, we have two comments to make.

It would be interesting to know for which measurable sets $A(x)$, the truncated Hilbert kernel $\frac{1}{t} \chi_{t \in A(x)}$ gives an operator bounded on $L^{p}$. The results in this paper answer this question for sets $A(x)=\{t: a|x|+b \leq t \leq c|x|+d\}$ and $A(x)=\{t: a x+b \leq t \leq c x+d\}$.

The big question is whether the bilinear Hilbert transform

$$
H(g, f)(x)=\text { p.v. } \int_{-\infty}^{\infty} g(x+t) f(x-t) \frac{d t}{t}
$$

maps $L^{\infty} \times L^{p}$ into $L^{p}$, for $1<p<+\infty$. As of this writing, M. Lacey and C. Thiele report significant progress on this problem.

\section{References}

[G] L. Grafakos, On multilinear fractional integrals, Studia Math., 102 (1992), 49-56. 
[HLP] G. Hardy, J. Littlewood and G. Pólya, Inequalities, Cambridge University Press, Cambridge, UK, 1934.

[SoW] F. Soria and G. Weiss, A remark on singular integrals and power weights, Indiana J. Math, 43 (1994), 187-204.

[S] E. M. Stein, Singular integrals and differentiability properties of functions, Princeton University Press, Princeton NJ, 1970.

[StW] E. M. Stein and G. Weiss, Introduction to Fourier Analysis on Euclidean spaces, Princeton University Press, Princeton NJ, 1971.

[Z] A. Zygmund, Trigonometric Series, Volume II, Cambridge Univ. Press, 2nd Ed., Cambridge, UK, 1959. 